\title{
Hygiene und Arbeitsschutz
}

\author{
S. Beckers
}

\subsection{Hygiene -41}

\section{$2.2 \quad$ Impfungen -48}

2.3 Arbeitsschutz -50

2.4 Meldepflichtige Erkrankungen - 53

$2.5 \quad$ Infektionstransport -54

Literatur - 57

Weiterführende Internetadressen - 57

\subsection{Hygiene}

Im Rettungsdienst werden unter dem Begriff »Hygiene « alle vorsorglichen Vorkehrungen und Maßnahmen zusammengefasst, die alle im Krankentransport und Rettungsdienst Tätigen, sowie die $\mathrm{zu}$ betreuenden Patienten, vor schädlichen und krankmachenden Einflüssen durch Mikroorganismen schützen sollen.

Ziel der Maßnahmen muss generell sein, im Idealfall eine Übertragung von Krankheitserregern auf den Patienten, das beteiligte Personal sowie deren Kontaktpersonen, z. B. Familienangehörige, zu verhindern oder dies größtmöglich zu minimieren.

Jeder Patient im Geltungsbereich des Rettungsdienstes kann erwarten, dass er durch einen Transport nicht einem erhöhten Infektionsrisiko ausgesetzt wird. Insbesondere gilt dies für infektionsgefährdete Patienten, z. B. im Rahmen einer Immunsuppression.

Als Grundlage für die Hygiene im Rettungsdienst dienen Rechtsgrundlagen und Empfehlungen ( Übersicht).

\section{Rechtsgrundlagen und Empfehlungen}

- Richtlinie für Krankenhaushygiene und Infektionsprävention des Robert-Koch-Instituts

- Empfehlungen des Arbeitskreises »Krankenhaushygiene« der AWMF (Arbeitsgemeinschaft der Wissenschaftlichen Medizinischen Fachgesellschaften)

- Infektionsschutzgesetz (IfSG)

- Arbeitsschutzgesetz (ArbSchG)

- Gefahrstoffverordnung (GefStoffV)

- Biostoffverordnung (BioStoffV)

- Unfallverhütungsvorschriften »Grundsätze der Prävention« (BGV A 1), "Gesundheitsdienst» (BGV C 8) sowie dazugehörige BG-Regeln und technische Regeln, z. B. Technische Regeln für Biologische Arbeitsstoffe (TRBA 250)

- Bestimmungen verschiedener Landesrettungsdienstgesetze

Diese Leitlinien, Richtlinien und Empfehlungen sind grundsätzlich zwar nicht rechtsverbindlich. Es 
kann in begründeten Fällen von ihnen abgewichen werden. Jedoch kann sich eine Verbindlichkeit von Richtlinien aus einem Gesetz oder aus Verträgen ergeben.

Verstöße gegen Hygienevorschriften können als Behandlungsfehler gewertet werden, wie Urteile der Vergangenheit zeigen, für die dann Rettungsdienstträger und ggf. behandelnde Ärzte Rechenschaft ablegen müssen. Analog gilt dies für erforderliche Organisationspflichten im Hygienebereich.

Gleichwohl gibt es im Hinblick auf die Hygienemaßnahmen im Rettungsdienst einige Problemfelder.

\section{Problemfelder bei rettungsdienstlichen} Hygienemaßnahmen

- Patiententransport bei unerkannter infektiöser Gefährdung

- Patiententransport bei bekanntem infektiösem Krankheitsbild

- Schnellstmögliche Wiederherstellung der Einsatzbereitschaft nach Transportbeendigung

- Aufbewahrung und Pflege der rettungstechnischen, pflegerischen und medizinischen Ausstattung auf begrenztem Raum und unter schwierigen hygienischen Bedingungen

- Spannungsfeld zwischen Priorisierung vitaler Funktionen gegenüber elementaren Grundregeln der Hygiene

\section{Allgemeine Hygiene- und Vorsichtsmaß- nahmen}

Für alle Mitarbeiter im Krankentransport und Rettungsdienst gilt, wie allgemein für medizinisches Personal, dass im Rahmen der Dispositionsprophylaxe alle Möglichkeiten der aktiven Immunisierung ausgeschöpft werden, um prinzipiell vermeidbare Infektionsrisiken auszuschalten (Aktuelle Empfehlungen nach STIKO - Abschn. »Impfungen«).

Um den Anforderungen an Hygiene im Rettungsdienstalltag gerecht zu werden, sind folgende
Ausstattungsmerkmale auf den Rettungsmitteln generell vorzuhalten:

- Händedesinfektionsmittel, alkoholische Lösung (nach Möglichkeit über einen Wandspender)

- Sterile und unsterile Einmalhandschuhe sowie robuste Arbeitshandschuhe (schnitt- und bissfest)

- Augenschutzbrille und Gesichtsmaske, ggf. mit Schutzschild

- Atemhilfsmittel bei Notbeatmung (z. B. für Mund-Gesichts-Maskenbeatmung)

- Abwurfbehälter für verletzende Gegenstände (Kanülen, Skalpell, Ampullen etc.)

- Papierhandtücher, Flächendesinfektionsmittel, saugfähiges Material (z. B. Zellstoff)

- Infektionsschutzset: Einmalwäsche, Einmalschutzkittel, Einmalhandschuhe, Mund-Nasen-Schutz, Haarschutz

Weiterhin ist es hilfreich, auch folgende Materialien jederzeit im Notfallkoffer bereitzuhalten:

- Händedesinfektionsmittel, alkoholische Lösung, ggf. einzeln verpackte Händedesinfektionstücher

- Sterile und unsterile Einmalhandschuhe

- Augenschutzbrille und Gesichtsmaske, ggf. mit Schutzschild

- Abwurfbehälter für verletzende Gegenstände (Kanülen, Skalpell, Ampullen etc.)

\section{Persönliche Hygiene des Rettungsdienstpersonals}

Unter persönlicher Hygiene werden alle Maßnahmen zusammengefasst, die dem Schutz der eigenen Person dienen, sowie im Rahmen der allgemeinen Körperpflege und täglichen Hygiene außerhalb des Arbeitsbereiches vorausgesetzt werden. Hierfür gilt es, folgende Maßnahmen generell oder situationsbedingt zu berücksichtigen:

- Tägliches Waschen oder Duschen

- Mehrmalige Haarwäsche und Haarpflege pro Woche

- Regelmäßige Pflege der Hände (z. B. durch spezielle Handpflegemittel), um eine erhöhte Übertragungsgefahr durch Risse zu verhindern 
- Fingernägel kurz und rund schneiden und nicht lackieren

- Kein Tragen von Schmuck (auch Armbanduhren) und Fingerringe wegen Verletzungsgefahr und drohender Keimübertragung

Für die tägliche Arbeit sind folgende Maßnahmen unerlässlich, wobei auf die entsprechende Einhaltung im Sinne einer gegenseitigen kollegialen Fürsorgepflicht geachtet werden sollte:

- Obligates Tragen von Berufs- bzw. Schutzkleidung (s. unten)

- Regelmäßiger Wechsel der Dienstkleidung bzw. nach jeder Kontamination im Sinne einer sichtbaren Verunreinigung

- Schuhe müssen den Sicherheitsvorschriften der UVV entsprechen und sollen leicht zu reinigen und an ihrer Oberfläche zu desinfizieren sein

- Bei Tätigkeiten, bei denen eine Kontamination möglich ist, d. h. bei jedem Patientenkontakt, sind zum Eigenschutz Einmalhandschuhe zu tragen

- Regelmäßige Anwendung der hygienischen Händedesinfektion

- Verwendung flüssigkeitsdichter Schutzbezüge für Tragen, Vakuummatratzen etc.

- Desinfektion der Standardgeräte Staubinde, Blutdruckmanschette sowie Stethoskop

- Verwendung von Einmalartikeln

- Regelmäßige Fahrzeugreinigung

\section{Hygienische Händedesinfektion}

\section{(!) Wichtig}

Die hygienische Händedesinfektion dient sowohl dem Schutz des Patienten als auch dem Eigenschutz und ist die wichtigste Maßnahme, um eine Übertragung von Infektionserregern zu verhindern.

Die hygienische Händedesinfektion bewirkt, dass die sog. transiente Hautflora (= nicht hauteigene Flora, vorübergehende Besiedelung mit z. B. pathogenen Keimen) gezielt reduziert wird. So können sich Patienten und Helfer sicher vor einer Übertragung von Infektionserregern über die Hände schützen.

\section{Vorgehen bei der hygienischen Händedesinfektion}

- Alkoholisches Händedesinfektionsmittel (ca. $3 \mathrm{ml}=2-3$ Hübe aus Wandspender, entspricht einer Hohlhand voll) in die komplett trockene und seifenfreie Hohlhand geben. Einwirkzeit von mindestens 60 s einhalten (Herstellerangaben einhalten!).

1. Verteilen des Desinfektionsmittels auf beiden Handflächen

2. Rechte Handfläche über linken Handrücken mit gespreizten Fingern reiben, dann umgekehrt

3. Handinnenflächen aufeinander legen und mit verschränkten, gespreizten Fingern verreiben

4. Handinnenflächen aufeinander legen, Finger miteinander verschränken und Fingerendglieder aneinander reiben

5. Umgreifen und kreisendes Reiben des Daumen mit der jeweils anderen Hand

6. Aneinanderlegen der Fingerkuppen und kreisendes Reiben hin und her in der jeweils anderen Hohlhand

- Wiederholung der Schritte 1-6 insgesamt 5mal, so lange bis die Einwirkzeit erreicht ist. Diesbezüglich unbedingt Herstellerangaben beachten!

Eine ordnungsgemäße Händedesinfektion und anschließende Waschung ist zu folgenden Zeiten erforderlich:

- Vor Arbeitsbeginn und nach Arbeitsende

- Nach jedem WC-Besuch

- Vor invasiven Eingriffen (z. B. Legen eines i.v.Zugangs, Blasenkatheters, Thoraxdrainage). Beachte: Gilt auch dann, wenn beim Eingriff an sich sterile Handschuhe getragen werden müssen!

- Vor Kontakt mit immunschwachen oder immunsuppremierten Patienten (z. B. bei Leukämie, Bestrahlungs- oder Intensivpatienten o. Ä.)

- Vor dem Betreten von Intensiv-, Infektionsoder Isolierstationen sowie OP-Bereichen

- Vor und nach jeder pflegerischen Versorgung

- Vor und nach jedem Anlegen eines Verbandes 
- Vor und nach Kontakt mit Eintrittsstellen von Kathetern, Drainagen o. Ä.

- Nach Schmutzarbeiten bzw. nach Kontakt mit Blut, Urin, Stuhl, Schleim etc.

- Nach Kontakt mit Patienten, die als potentiell infektiös gelten

- Nach Kontakt mit kontaminierten Flächen oder Gegenständen (z. B. Beatmungszubehör, Steckbecken, Arbeitsflächen)

\section{Waschen der Hände}

Das Waschen der Hände ist generell indiziert

- Vor Arbeitsbeginn und nach Arbeitsende

- Nach längeren Pausen

- Bei sichtbarer Verschmutzung ohne bestehende Infektionsgefahr

Im Falle einer sichtbaren Verschmutzung mit potentiell möglicher Infektionsgefahr sollte erst eine hygienische Händedesinfektion (s. oben) erfolgen, dann die Handwaschung (unbedingt Hände abtrocknen!) und ggf. eine erneute Desinfektion durchgeführt werden.

Wenn möglich sollte jeweils zur Vorbereitung einer hygienischen Händedesinfektion die Hände mit Flüssigseifen gewaschen werden. Zum Abtrocknen dürfen ausschließlich Einmalhandtücher verwendet werden. Allerdings kann durch zu häufiges Händewaschen die Haut sehr trocken und spröde werden. Auch Hautrisse können auftreten.

\section{Pflege der Hände}

Eine Pflege der Hände sollte als selbstverständlich gelten, so dass auf eine regelmäßige Pflege und nach Bedarf, z. B. vor längeren Pausen und nach Arbeitsende, unter Verwendung von entsprechenden Pflegeprodukten zu achten ist.

\section{(D) Wichtig}

Kleinste Hautrisse sind Reservoire für Infektionserreger und somit potentielle Infektionsquellen! Adäquate Hautpflege ist daher unerlässlich und beugt Hautschäden und Infektionen vor!

Neben dem konsequenten Tragen von Schutzhandschuhen beim Umgang mit Patienten bietet die Erhaltung einer intakten und gesunden Haut einen optimalen Infektionsschutz. Um dies zu erreichen, sollten regelmäßig Hautschutz- und Pflegeprodukte angewendet werden. Weitere Empfehlungen:

- Hautpflegemittel aus Tube oder Spender entnehmen und wegen erhöhter Kontaminationsgefahr nicht aus Dosen oder Salbentöpfen

- Schutzhandschuhe nur auf trockener Haut tragen

- Alkoholische Händedesinfektion nur auf trockener Haut anwenden

- Vermischung von Desinfektionsmittel und Seife vermeiden

Die entsprechenden Maßnahmen und zur Verfügung stehenden Pflegemittel müssen in jeder Rettungswache, d. h. an jedem Handwaschplatz, in einem Hautpflegeplan zusammengestellt sein.

\section{Ratschläge für die persönliche Hygiene}

- Während der Händedesinfektion das Desinfektionsmittel nicht auf die nassen Hände geben!

- Sichtbare Verschmutzungen der Hände sind durch Waschen zu entfernen (Wasser nicht zu warm, Seifenreste komplett abspülen, Haut sorgfältig trocknen)

- Mindesteinwirkzeit von $30 \mathrm{~s}$ reicht nicht aus, um einige Erreger (z. B. Pseudomonas, unbehüllte Viren) auszuschalten!

- An Händen und Unterarmen dürfen keine Schmuckstücke, z. B. Eheringe, und Uhren getragen werden. Sie behindern die hygienische Händedesinfektion, weil Handgelenke ebenfalls desinfiziert werden müssen. Außerdem können sie bei der Versorgung von Patienten zu Verletzungen führen!

- Nagellack, lange sowie künstliche Fingernägel beeinträchtigen die Wirkung der Händedesinfektion und sind nach TRBA 250 verboten!

\section{Standard-Hygienemaßnahmen im Umgang mit Patienten}

Hierunter versteht man alle Maßnahmen der Infektionskontrolle, die im Umgang mit allen Pati- 
enten berücksichtigt werden sollen. Dies geschieht unabhängig davon, ob tatsächlich eine Infektion vorliegt bzw. bekannt ist oder nicht. Diese Hygienemaßnahmen bieten zudem bei den meisten Infektionen einen ausreichenden Schutz:

- Händehygiene, Händewaschen/Händedesinfektion nach Kontamination bzw. vor Tätigkeiten, bei denen der Patient vor Kontamination zu schützen ist (z. B. invasiven Maßnahmen)

- Tragen von Einmalhandschuhen bei jedem Patientenkontakt, sowie bei Kontakt mit Sekreten, Exkreten, Blut, Stuhl etc.

- Verwendung von Schutzkleidung zusätzlich zur Arbeitskleidung, wenn eine Kontamination mit potentiell infektiösem Material möglich ist

- Schutz der Schleimhäute (Mund-/Nasenschutz ggf. Augenschutz), um Kontakt mit potentiell infektiösem Material (z. B. Blut, respiratorisches Sekret etc.) zu vermeiden

- Reinigung sowie Desinfektion und ggf. Sterilisation von Instrumenten und Gegenständen der Patientenversorgung (z. B. Blutdruckmanschette, Staubinde etc.)

- Gezielte Flächendesinfektion (im Wischverfahren) nach Kontamination sowie bei ausgedehnter Kontamination des Rettungsmittels

- Entsorgung potentiell verletzender Gegenstände unmittelbar nach Gebrauch in Sicherheits-Abwurfbehälter.

\section{(?) Wichtig}

Folgende Überlegungen zu Hygienemaßnahmen sind besonders hervorzuheben und in den jeweiligen Situationen zu berücksichtigen:

- Korrekte Hautdesinfektion vor Injektionen oder Anlage von i.v.-Zugängen

- Sterile Hautabdeckung vor invasiven Eingriffen (z. B. Bülaudrainage) mit Klebefolien

- Steriles Abwaschen vor der Anlage von Harnblasenkathetern

- Sterile Abdeckung von Verbrennungen, offenen Frakturen oder anderen Wunden

- Verwendung steriler Handschuhe und Mund-Nasen-Schutz bei Patienten mit bekannter Immunschwäche oder bei Immunsuppression

\section{Nadelstichverletzungen}

Um Arbeitsunfällen vorzubeugen, ist die Entsorgung potentiell verletzender Gebrauchsgegenstände unmittelbar nach Verwendung in dafür vorgesehene Sicherheits-Abwurfbehälter sehr wichtig.

Folgende Umstände oder Verhaltensweisen sind im rettungsdienstlichen Alltag zudem als äußerst riskant einzustufen:

- Mangelhafte oder überfüllte Entsorgungsbehälter

- Unzureichende Entsorgung gebrauchter Kanülen oder Instrumente

- Manuelles Entfernen der Kanüle von einer Spritze

- Fremdverschulden (z. B. durch Bewegung der Patienten oder Fahrzeugbewegung)

\section{(D) Wichtig}

Die häufigsten Arbeitsunfälle im medizinischen Bereich sind Nadelstichverletzungen, vor allen Dingen durch Zurückstecken einer Kanüle in die Hülle, dem sog. »recapping«.

\section{Exposition mit potentiell HIV-haltigem Material}

Im Falle einer Nadelstichverletzung bei unklarem Infektionsstatus des Patienten, insbesondere nach HIV-Exposition, empfiehlt das Robert-Koch-Institut folgendes Vorgehen, sog. HIV-Postexpositionsprophylaxe (HIV-PEP):

- Förderung bzw. Induzierung eines Blutflusses, z. B. durch Druck auf umliegendes Gewebe für $\geq 1 \min$

- Ausgiebige antiseptische Spülung oder Anlage eines antiseptischen Wirkstoffdepots (z. B. in Form von mit Antiseptikum getränkten Tupfern)

- Meldung und Vorstellung bei einem D-Arzt zur Unfalldokumentation und Einleitung eines BG-Verfahrens

- Ggf. systemische, medikamentöse Postexpositionsprophylaxe

- Erste Testung auf HIV-Antikörper und Hepatitisserologie

Neben der Gefährdung durch Nadelstichverletzung sollten nach HIV-Exposition ebenfalls So- 
fortmaßnahmen in folgenden Situationen ergriffen werden:

- Exposition geschädigter Haut

- Spritzer ins Auge

- Spritzer in die Mundhöhle

Eine spezielle HIV-PEP sollte frühestmöglich in die Wege geleitet werden, d. h. gemäß den Empfehlungen des RKI innerhalb von $24 \mathrm{~h}$ nach Exposition; innerhalb von $2 \mathrm{~h}$ nach Exposition ist mit den besten Ergebnissen zu rechnen. Nach einem Zeitraum von mehr als $72 \mathrm{~h}$ wird die Durchführung einer HIV-PEP nicht mehr empfohlen. Aufgrund des zeitlich sehr eingeschränkten Behandlungskorridors ist es von Seiten jeder ärztlichen Leitung Rettungsdienst sinnvoll, eine für diesen Fall ausgearbeitete Handlungsanweisung mit Angabe der Indikationen, praktischer Vorgehensweise für die medikamentöse PEP, sowie jederzeit erreichbare Ansprechpartner zu erstellen und allgemein zugänglich zu hinterlegen. Weiterhin sollte wegen der zeitkritischen Komponente eine zentrale Vorhaltung - abhängig von der Struktur des Rettungsdienstes, z. B. Rettungswache, Notaufnahme etc. - aktuell empfohlener Medikamente organisiert sein sowie eine regelmäßige Schulung aller Rettungsdienstmitarbeiter zu diesem Themenkomplex stattfinden.

Zudem wird bereits im Rettungsdienst ein »Nadelstich-Sofortmaßnahmen-Set « als Ergänzung der RTW-Ausstattung vorgehalten, damit umgehend erforderliche Maßnahmen wie etwa Augenoder Mundspülung, Erweitern des Stichkanals etc. durchgeführt werden können. Inhalt des »Nadelstich-Sofortmaßnahmen-Set« sind:

- Zusammenfassung der Empfehlungen gemäß RKI und Darstellung der lokalen Vorgehensweise

- 50-ml-Spritze

- Einmal-Skalpell

- (Mess-)Becher

- Ethanol- und jodhaltiges Desinfektionsmittel

- Wasser zur Verdünnung

Einen Überblick über die Indikationen zur HIVPEP bei beruflicher HIV-Exposition gemäß RKI gibt - Tab. 2.1.

\section{Hepatitis-B-Immunprophylaxe bei Exposition mit HBV-haltigem Material}

Als HBV-haltig wird HBsAg-positives Material bezeichnet bzw. wahrscheinlich kontaminiertes Material, bei dem eine Testung nicht möglich ist, z. B. mit Blut kontaminierte Punktionskanüle im Abwurfbehälter.

Für geimpfte Personen wird folgendes, angepasstes Vorgehen empfohlen:

- Keine Maßnahmen erforderlich bei

- nicht länger als 5 Jahre zurückliegender Impfung und einem Anti-HBs von $\geq 100$ IE/l nach Grundimmunisierung bei der exponierten Person,

- einem Anti-HBs-Wert $\geq 100$ IE/l innerhalb der letzten 12 Monate unabhängig vom Zeitpunkt der Grundimmunisierung.

- Tab. 2.1. Indikation zur HIV-PEP bei beruflicher HIV-Exposition

\begin{tabular}{l|c}
\hline $\begin{array}{l}\text { Perkutane Verletzung mit Injektionsnadel oder anderer Hohlraumnadel (Körperflüssigkeit mit } \\
\text { hoher Viruskonzentration: Blut, Liquor, Punktatmaterial, Organmaterial) }\end{array}$ & Empfehlen \\
\hline Oberflächliche Verletzung (z. B. mit chirurgischer Nadel) & Anbieten \\
\hline Ausnahme: Indexpatient hat Aids oder eine hohe HI-Viruskonzentration & Empfehlen \\
\hline $\begin{array}{l}\text { Kontakt zu Schleimhaut oder verletzter/geschädigter Haut mit Flüssigkeiten mit hoher Virus- } \\
\text { konzentration }\end{array}$ & Nicht empfehlen \\
\hline Perkutaner Kontakt mit anderen Körperflüssigkeiten als Blut (wie Urin oder Speichel) & Nicht empfehlen \\
\hline Kontakt von intakter Haut mit Blut (auch bei hoher Viruskonzentration) & Nicht empfehlen
\end{tabular}


- Umgehende Verabreichung von Hepatitis-BImpfstoff (ohne weitere Maßnahmen) bei

- einer bereits 5-10 Jahre zurückliegenden Impfung,

- auch wenn der initiale Anti-HBs $\geq 100$ IE/l nach Grundimmunisierung war.

- Umgehende Testung der exponierten Person (»Empfänger«) bei

- nicht bzw. nicht vollständig geimpften Empfänger,

- »Low respondern«, d. h. Personen mit einem Anti-HBs $<100$ IE/l nach Grundimmunisierung,

- unzureichender oder nicht stattgefundener Kontrolle des Impferfolges,

- länger als 10 Jahre zurückliegender Impfung.

Abhängig vom Testergebnis werden in diesem Fall HB-Impfstoff und/oder HB-Immunglobulin appliziert (•Tab. 2.2).

"Non-Responder«, d.h. Personen mit einem Anti-HBs $<10$ IE/l nach drei oder mehr Impfungen sowie Anti-HBs-Negative erhalten nach Exposition unverzüglich HB-Impfstoff und HB-Immunglobulin.

\section{Hygiene im Umgang mit Medizingeräten und Verbrauchsmaterial}

Bei der Hygiene der im Rettungsdienst und Krankentransport eingesetzten Medizingeräte sind folgende Grundsätze zu beachten:

- Für den Einmalgebrauch bestimmtes Verbrauchsmaterial ist bestimmungsgemäß zu entsorgen oder ggf. nach Herstellerangaben aufzubereiten!

- Geräteoberflächen können unter Berücksichtigung der Herstellerangaben wischdesinfiziert werden!

- Medizinische Instrumente sollten nach Gebrauch im Fahrzeug in verschließbare Behälter verwahrt werden und ggf. in der Rettungswache in dafür vorgesehenen Behältnissen desinfiziert werden!

- Generell sind Einmaltragebezüge und -wäsche zu bevorzugen!

Eine Abfallentsorgung kann nach Aufteilung in übliche Fraktionen gemäß Vorschrift entsorgt werden. Folgende Abfallgruppen werden unterschieden:

- A - Hausmüll

- B - Mit Blut, Sekreten

- C - Abfall aus Isolierstationen

- D - Umweltbelastende Chemikalien

- E - Organe und Organteile

\section{Wichtig}

Generell sollten am Einsatzort keine Abfälle zurückbleiben. Insbesondere spitze und scharfe Gegenstände sind unverzüglich und sicher zu entsorgen.

\section{Hygiene der Rettungsmittel}

Folgende Richtwerte für die Reinigung bzw. Desinfektion von Rettungsmitteln haben sich im Einsatzalltag bewährt, wobei gebrauchsfertig angesetzte Desinfektionslösungen in handlichen Spritzfla-

- Tab. 2.2. Hepatitis-B-Prophylaxe nach Exposition und Testung

\begin{tabular}{l|l|l}
\hline $\begin{array}{l}\text { Aktueller Anti-HBs-Wert } \\
\text { [IE/I] }\end{array}$ & Erforderlich ist die Gabe von & HB-Immunglobulin \\
\hline$\geq 100$ & HB-Impfstoff & Nein \\
\hline$\geq 10$ bis $<100$ & Nein & Nein \\
\hline$<10$ & Ja & Ja \\
\hline Nicht innerhalb von $48 \mathrm{~h}$ & Ja & Ja \\
\hline
\end{tabular}


schen - eindeutig gekennzeichnet und mit Haltbarkeitsdatum - gemäß Desinfektionsmittelliste verwendet werden:

- Gezielte Flächendesinfektion unmittelbar nach Kontamination mit potentiell infektiösem Material und/oder nach Transport von potentiell infizierten Patienten (s. unten)

- In der Regel Scheuer-Wisch-Desinfektion mit aldehydischen Flächendesinfektionsmittel in der für Krankenhäuser üblichen Konzentration (DGHM-Liste)

- Gründliche Lüftung des Fahrzeugs nach jeder Flächendesinfektion!

- Tägliche Oberflächenreinigung ohne Desinfektionsmittel (einschließlich Fußboden) zur Entfernung normaler Verschmutzung

- Wöchentliche Grundreinigung des Patientenraumes, sowie der Fahrerkabine und der Ausstattung

\section{Transportübernahme}

Im Rahmen eines Notfalleinsatzes sind dem Rettungsdienstpersonal bereits vorliegende Informationen hinsichtlich eines erhöhten Infektionsrisikos vor der Versorgung des Patienten und einem möglichen Transport mitzuteilen. Da dies in der größten Zahl der Einsätze nicht der Fall sein wird, ist im Zweifel von einer infektiösen Gefährdung auszugehen und die Standardhygiene- und Vorsichtsmaßnahmen strikt zu berücksichtigen (Details s. oben).

Sind im Vorfeld der Übernahme eines Sekundärtransportes Erkrankungen mit erhöhtem Infektionsrisiko bekannt, so sind diese der zuständigen Rettungsleitstelle bei Anforderung des Transportes bereits mitzuteilen, damit etwaige notwendige Hygiene- und Vorsichtsmaßnahmen im Vorfeld getroffen werden können, z. B. Entsendung eines speziell vorgehaltenen Rettungsmittels für Infektionstransporte.

\subsection{Impfungen}

Für alle im Krankentransport und Rettungsdienst tätigen Mitarbeiter ist es wichtig, auf einen vollständigen Impfschutz zu achten. Grundlage hierfür sind die gültigen Empfehlungen der Ständi- gen Impfkommission am Robert-Koch-Institut (STIKO) mit derzeitigem Stand vom Juli 2006, die als sog. "Epidemiologisches Bulletin« auf den Internetseiten des Institutes in der Rubrik »Infektionsschutz« zur Verfügung stehen.

\section{(? Wichtig}

Ein adäquater Impfschutz gegen Hepatitis B und Tetanus ist im Rettungsdienst essentiell! Empfohlen wird zudem ein ausreichender Impfschutz gegen Diphtherie, Haemophilus influenzae, Pertussis und Röteln!

\section{Impfkalender}

In - Tab. 2.3 ist der aktuell von der STIKO empfohlene Impfkalender für Säuglinge, Kinder, Jugendliche und Erwachsene dargestellt.

Die dargestellten Standardimpfungen des Impfkalenders (S) sind gemäß STIKO »von hohem Wert für den Gesundheitsschutz des Einzelnen und der Allgemeinheit«. Sie werden deshalb auch für alle Angehörigen der jeweiligen Alters- oder Bevölkerungsgruppen empfohlen.

Darüber hinaus werden weitere Impfungen in der Kategorie B, d. h. aufgrund eines erhöhten beruflichen Risikos, aufgeführt und treffen ggf. auch für die Berufsgruppe der im Rettungsdienst tätigen Mitarbeiter zu.

\section{Empfehlung für Impfungen in der Kategorie B (Auszug STIKO)}

- Hepatitis A (HA): sog. HA-gefährdetes Personal (medizinisches und anderes Fach- und Pflegepersonal) im Gesundheitsdienst, z. B. Pädiatrie und Infektionsmedizin

- Hepatitis B (HB): HB-gefährdete Personen im Gesundheitsdienst einschließlich Auszubildender bzw. Studenten sowie Hersteller; Reinigungspersonal

- Influenza: Personen mit erhöhter Gefährdung, z. B. medizinisches Personal, Personen in Einrichtungen mit umfangreichem Publikumsverkehr sowie Personen, die als mögliche Infektionsquelle für von ihnen betreute ungeimpfte Risikopersonen fungieren können 
- Tab. 2.3. Impfkalender (Standardimpfungen) für Säuglinge, Kinder, Jugendliche und Erwachsene. Empfohlenes Impfalter und Mindestabstände zwischen den Impfungen

\begin{tabular}{|c|c|c|c|c|c|c|c|c|c|c|}
\hline \multirow{2}{*}{$\begin{array}{l}\text { Impfstoff/Antigen- } \\
\text { Kombinationen }\end{array}$} & \multicolumn{6}{|c|}{ Alter in vollendeten Monaten } & \multicolumn{4}{|c|}{ Alter in vollendeten Jahren } \\
\hline & Geburt & 2 & 3 & 4 & $11-14$ & $\begin{array}{l}15-23 \\
\text { siehe a) }\end{array}$ & $\begin{array}{l}5-6 \\
\text { siehe a) }\end{array}$ & $\begin{array}{l}\text { 9-17 } \\
\text { siehe a) }\end{array}$ & ab 18 & $\geq 60$ \\
\hline$T^{*}$ & & 1. & 2. & 3. & 4. & & $A$ & A & $A^{* * * * *}$ & \\
\hline$D / d *$ siehe $b$ ) & & 1. & 2. & 3. & 4. & & $A$ & $A$ & $A^{* * * * *}$ & \\
\hline aP/ap * & & 1. & 2. & 3. & 4. & & A & A & & \\
\hline $\mathrm{Hib}^{*}$ & & 1. & 2. c) & 3. & 4. & & & & & \\
\hline IPV * & & 1. & 2.c) & 3. & 4. & & & A & & \\
\hline$H B^{*}$ & d) & 1. & 2. c) & 3. & 4. & & & G & & \\
\hline Pneumokokken** & & 1. & 2. & 3. & 4. & & & & & S \\
\hline Meningokokken & & & & & $\begin{array}{l}\text { 1.e) ab vollende- } \\
\text { tem } 12 \text {. Monat }\end{array}$ & & & & & \\
\hline$M M R * * *$ & & & & & 1. & 2. & & & & \\
\hline Varizellen & & & & & 1. & f) & & g) & & \\
\hline Influenza **** & & & & & & & & & & $S$ \\
\hline
\end{tabular}

Legende und Hinweise STIKO: Um die Zahl der Injektionen möglichst gering zu halten, sollten vorzugsweise Kombinationsimpfstoffe verwendet werden. Impfstoffe mit unterschiedlichen Antigenkombinationen von D/d, T, aP/ap, HB, Hib, IPV sind verfügbar. Bei Verwendung von Kombinationsimpfstoffen sind die Angaben des Herstellers zum Impfalter und zu den Impfabständen zu beachten.

A: Auffrischimpfung: Diese sollte möglichst nicht früher als 5 Jahre nach der vorhergehenden letzten Dosis erfolgen.

G: Grundimmunisierung aller noch nicht geimpften Jugendlichen bzw. Komplettierung eines unvollständigen Impfschutzes.

S: Standardimpfungen mit allgemeiner Anwendung = Regelimpfungen .

a. Zu diesen Zeitpunkten soll der Impfstatus unbedingt überprüft und ggf. vervollständigt werden.

b. Ab einem Alter von 5 bzw. 6 Jahren wird zur Auffrischimpfung ein Impfstoff mit reduziertem Diphtherietoxoid-Gehalt (d) verwendet.

c. Bei monovalenter Anwendung bzw. bei Kombinationsimpfstoffen ohne Pertussiskomponente kann diese Dosis entfallen.

d. Siehe Anmerkungen »Postexpositionelle Hepatitis-B-Immunprophylaxe bei Neugeborenen« (S. 237, der STIKO-Empfehlung 30/2006).

e. Der Meningokokken-Konjugatimpfstoff sollte nicht gleichzeitig mit Pneumokokken-Konjugatimpfstoff oder MMR- und Varizellen-Impfstoff oder MMRV gegeben werden. Siehe auch »Anmerkungen zu den im Impfkalender aufgeführten Impfungen«

(S. 238 der STIKO-Empfehlung 30/2006).

f. Bei Anwendung des Kombinationsimpfstoffes MMRV sind die Angaben des Herstellers zu beachten. Entsprechend den Fachinformationen ist die Gabe einer 2. Dosis gegen Varizellen erforderlich. Zwischen beiden Dosen sollten 4-6 Wochen liegen.

g. Weitere Details siehe Tab. 2 der STIKO-Empfehlung 30/2006.

* Abstände zwischen den Impfungen mindestens 4 Wochen; Abstand zwischen vorletzter und letzter Impfung mindestens 6 Monate.

** Generelle Impfung gegen Pneumokokken für Säuglinge und Kleinkinder bis zum vollendeten 2. Lebensjahr mit einem Pneumokokken-Konjugatimpfstoff; Standardimpfung für Personen - 60 mit Polysaccharid-Impfstoff und Wiederimpfung im Abstand von 6 Jahren.

*** Mindestabstand zwischen den Impfungen 4 Wochen.

**** Jährlich mit dem von der WHO empfohlenen aktuellen Impfstoff.

***** Jeweils 10 Jahre nach der letzten vorangegangenen Dosis. 
- Poliomyelitis:

- Personal der oben genannten Einrichtungen

- Medizinisches Personal, das engen Kontakt zu Erkrankten haben kann

- Personal in Laboratorien

- Varizellen: Seronegatives Personal im Gesundheitsdienst, insbesondere in:

- Pädiatrie

- Onkologie

- Gynäkologie/Geburtshilfe

- Intensivmedizin

- Bereich der Betreuung von Immundefizienten

- Bei Neueinstellungen in Gemeinschaftseinrichtungen für das Vorschulalter

\subsection{Arbeitsschutz}

\section{Persönliche Schutzausrüstung (PSA)}

\section{Zuständigkeit und Grundsätzliches}

Jeder im Rettungsdienst tätige »Unternehmer« (Hilfsorganisationen, private oder öffentliche Rettungsdienste) ist dazu verpflichtet, seinen Mitar- beitern eine persönliche Schutzausrüstung (PSA) in ausreichender Anzahl kostenlos zur Verfügung $\mathrm{zu}$ stellen (GUV-V A $1 \$ 29$, GUV-R 250 Abschnitt 4.1.3), sowie für deren Instandhaltung, Reinigung und Desinfektion zu sorgen (GUV-R 250, Abschnitt 4.1.3, \$3 Abs. 3 ArbSchG). Die entsprechende Ausrüstung muss dem Versicherten individuell passen und sollte damit grundsätzlich nur für den Gebrauch durch Einzelpersonen vorgesehen sein. Auf der anderen Seite sind die Mitarbeiter gemäß verschiedener Vorschriften auch dazu verpflichtet, die zur Verfügung gestellte PSA zu benutzen ( $\$ 30$ GUV-V A $1, \S 15$ ArbSchG).

Da die Beschaffenheit der jeweiligen Schutzausrüstung von den an der Arbeits- bzw. Einsatzstelle möglichen Gefahren abhängig ist, sollte eine PSA im Rettungsdienst insbesondere vor folgenden $\mathrm{Ge}$ fahren schützen (- Tab. 2.5):

- Gefahren bei Tätigkeiten im öffentlichen Verkehrsraum

- Klimatische Einwirkungen, z. B. Einwirkung von Nässe, Wind und Umgebungskälte

- Mechanische Einwirkungen unterschiedlichster Art

- Infektionen, d. h. Schutz vor Krankheitserregern und gesundheitsschädigenden Stoffen

Tab. 2.4. Tetanus-Immunprophylaxe im Verletzungsfall

\begin{tabular}{|c|c|c|c|c|}
\hline \multirow{2}{*}{$\begin{array}{l}\text { Vorgeschichte der Tetanus-Immunisierung } \\
\text { (Anzahl der Impfungen) }\end{array}$} & \multicolumn{2}{|c|}{ Saubere, geringfügige Wunden } & \multicolumn{2}{|c|}{ Alle anderen Wunden ${ }^{a}$} \\
\hline & $\mathrm{Td}^{\mathrm{b}}$ & $\mathrm{TIG}^{\mathrm{c}}$ & $\mathrm{Td}^{\mathrm{b}}$ & $\mathrm{TIG}^{\mathrm{c}}$ \\
\hline Unbekannt & $\mathrm{Ja}$ & Nein & Ja & $\mathrm{Ja}$ \\
\hline 0 bis 1 & $\mathrm{Ja}$ & Nein & Ja & $\mathrm{Ja}$ \\
\hline 2 & $\mathrm{Ja}$ & Nein & Ja & Nein ${ }^{d}$ \\
\hline 3 oder mehr & Nein ${ }^{e}$ & Nein & Nein ${ }^{d}$ & Nein \\
\hline
\end{tabular}

a Tiefe und/oder verschmutzte (mit Staub, Erde, Speichel, Stuhl kontaminierte) Wunden, Verletzungen mit Gewebszertrümmerung und reduzierter Sauerstoffversorgung oder Eindringen von Fremdkörpern (z. B. Quetsch-, Riss-, Biss-, Stich-, Schusswunden); schwere Verbrennungen und Erfrierungen, Gewebsnekrosen, septische Aborte.

${ }^{b}$ Kinder unter 6 Jahren T, ältere Personen Td (d. h. Tetanus-Diphtherie-Impfstoff mit verringertem Diphtherietoxoid-Gehalt). Jede Auffrischimpfung mit Td sollte Anlass sein, eine mögliche Indikation einer Pertussis-Impfung zu überprüfen und ggf. einen Kombinationsimpfstoff (Tdap) einzusetzen.

' TIG = Tetanus-Immunglobulin, im Allgemeinen werden 250 I.E. verabreicht, die Dosis kann auf 500 I.E. erhöht werden; TIG wird simultan mit Td/T-Impfstoff angewendet.

d Ja, wenn die Verletzung länger als $24 \mathrm{~h}$ zurückliegt.

e Ja (1 Dosis), wenn seit der letzten Impfung mehr als 10 Jahre vergangen sind.

f Ja (1 Dosis), wenn seit der letzten Impfung mehr als 5 Jahre vergangen sind. 
Aus der statistischen Auswertung von Arbeitsunfällen im Rettungsdienst geht hervor, dass die Extremitäten einem erhöhten Verletzungsrisiko ausgesetzt sind.

\section{Verteilung der Arbeitsunfälle im Rettungsdienst}

- Ein Drittel Hand- und Handgelenksverletzungen

- Ein Drittel Fußverletzungen

- Ein Drittel Rumpf- oder Kopf-Verletzungen bzw. ganzheitliche Gesundheitsschäden

Die Empfehlungen zur PSA im Rettungsdienst entstammen der GUV-Regel 2106, die sich vornehmlich an die sog. Unternehmer richtet und Hilfestellungen formuliert, die der Einhaltung arbeitsschutzrechtlicher Bestimmungen und Unfallverhütungsvorschriften dienen.

Zudem enthalten Sie Hinweise, wie Berufskrankheiten, Arbeitsunfälle und arbeitsbedingte Gesundheitsgefahren vermieden bzw. reduziert werden können. Die GUV-Regel 2106 gibt Auskunft über technische Einzelheiten, sowie entsprechende Verwendung der unterschiedlichen PSA in Notfallrettung und Krankentransport, wobei spezielle PSA z. B. für die Berg-, Wasser-, Schiffs- und Luftrettung sowie für die Rettung bei
Unfällen mit Radioaktivität, chemischen Stoffen oder bei technischer Hilfeleistung explizit ausgenommen sind.

Weitere Arten von PSA wie z. B. umluftabhängiger Atemschutz können im Bedarfsfall und je nach Gefährdung erforderlich werden.

\section{(. Wichtig}

Eine PSA, die universell gegen alle denkbaren Einwirkungen schützt, existiert nicht. Daher kann die Verwendung unterschiedlicher PSA - einzeln oder in Kombination - notwendig werden.

\section{Schutzkleidung}

Die zu erfüllenden Aufgaben einer Schutzkleidung im Rettungsdienst sind wie folgt beschrieben:

- Erkennen des Trägers beim Einsatz im Straßenverkehr in ausreichender Entfernung und bei Dunkelheit

- Schutz vor mechanischen und thermischen Einflüssen

- Schutz vor Witterungseinflüssen aller Art

- Schutz vor Einwirkung und unkontrollierter Verschleppung von Krankheitserregern

Um die Schutzwirkung zu gewährleisten, können normgerechte Jacken bzw. Kombinationen aus Jacke und Hose getragen werden. An die verwendete Hose werden nur dann keine besonderen Anfor-

- Tab. 2.5. Im Rettungsdienst und Krankentransport verwendete Arten von PSA

\begin{tabular}{l|l}
\hline Art & \multicolumn{1}{l}{ Gefährdung } \\
\hline Schutzkleidung & - Straßenverkehr \\
& - Krankheitserreger \\
& - Witterungseinflüsse (Nässe, Kälte) \\
\hline Handschutz & - Mechanische Einflüsse \\
& - Kontakt mit Krankheitserregern \\
\hline Fußschutz & - Verletzungen durch Umknicken, Ausrutschen, Vertreten \\
& - Mechanische Einflüsse \\
& - Chemische Einwirkungen \\
\hline Kopf-, Augen- und Gesichtsschutz & - Pendelnde, herabfallende, umfallende oder wegfliegende Gegenstände \\
& - Anstoßen \\
&
\end{tabular}


derungen (Warnwirkung und Hintergrundfarbe) gestellt, wenn die eingesetzte Jacke für sich der sog. Bekleidungsklasse 2 bzw. 3 nach DIN EN 471, entspricht.

Neben der Darstellung der zugelassenen Farben gemäß DIN EN 471 gibt die bereits erwähnte GUV-Regel 2106 auch detaillierte Empfehlungen über Art des erforderlichen retroflektierenden Materials und zeigt Beispiele für empfehlenswerte Ausführungen von Warnkleidung. Hinsichtlich der Anordnung von Reflexstreifen auf der Schutzkleidung wird die Kombination von waagerechten mit senkrechten Streifen empfohlen.

Zudem wird grundsätzlich darauf hingewiesen, dass die Schutzkleidung des Rettungsdienstes nicht vor Flammen oder großer Hitze (z. B. bei Rettung aus brennenden Objekten) schützt.

\section{Handschutz}

Pro Mitglied einer Fahrzeugbesatzung sollen auf jedem Rettungsmittel ein Paar Schutzhandschuhe nach DIN EN 659 »Feuerwehrschutzhandschuhe» vorgehalten werden, um im Bedarfsfall z. B. vor Schnittverletzungen durch Glassplitter oder scharfe Kanten zu schützen. Bei wechselnden Fahrzeugbesatzungen ist dann auf die Einhaltung entsprechender Hygienemaßnahmen, d. h. das Tragen von Einmalhandschuhen unter den Schutzhandschuhen, zu achten.

\section{Fußschutz}

Alle im Rettungsdienst und Krankentransport Tätigen sollen zum Schutz vor Verletzungen durch Umknicken, Ausrutschen, Vertreten sowie gegen mechanische oder chemische Einflüsse mindestens Sicherheitsschuhe der Kategorie S 2 Typ B (nach DIN EN 345) mit rutschhemmender Sohle und Zehenschutzkappe tragen. Darin enthalten sind u. a. auch die Forderung nach einem mindestens knöchelhohen Schaft, einem geschlossenen Fersenbereich, sowie nach Wasserdichtigkeit, Mittelfußschutz und einem anatomisch ausgebildeten Fußbett aufgeführt.

\section{Kopf-, Augen- und Gesichtsschutz}

Pro Mitglied einer Fahrzeugbesatzung ist auf jedem Rettungsmittel ein Schutzhelm nach DIN EN 443 »Feuerwehrhelme« mit Kinnriemen, Gesichts- und
Nackenschutz vorzuhalten. Dieser soll im entsprechenden Einsatzfall gegen Anstoßen des Kopfes, sowie vor pendelnden, herab- bzw. umfallenden oder wegfliegenden Gegenständen schützen. Der Gesichtsschutz (Visier) und Nackenschutz sind erforderlich, da es bei etwaigen Rettungseinsätzen zu Funkenflug kommen kann oder mit wegfliegenden Teilen gerechnet werden muss. Das individuelle Tragen von Helmen ist aus Gründen der Hygiene $\mathrm{zu}$ bevorzugen. Anderenfalls sollten bei Nutzung durch mehrere Personen Papierschonmützen unter dem Helm getragen werden.

Darüber hinaus sollten zum Augenschutz auf den Rettungsmitteln Schutzbrillen mit indirekter Belüftung und seitlichem Spritzschutz (z. B. nach DIN EN 166) vorgehalten werden, die z. B. gegen das Verspritzen infektiöser Flüssigkeit schützen.

\section{Infektionsschutz}

Entsprechend der Technischen Regel für Biologische Arbeitsstoffe (TRBA 250) werden Tätigkeiten im Krankentransport individuell vom Patienten abhängig in ihrer Gefährdung eingeteilt und das Vorgehen entsprechend angepasst. Eine detaillierte Darstellung findet sich in $>$ Abschn. »Infektionstra nsport«.

Die Tätigkeiten in der Notfallrettung werden gemäß TRBA 250 der sog. Schutzstufe 2, d.h. regelmäßiger Umgang mit infektiösem Material, ggf. 3, d. h. regelmäßiger Umgang mit Arbeitsstoffen, die als schwer krankheitserregend eingestuft werden, zugeordnet. Aus diesem Grund wird beim Umgang mit Patienten das Tragen von festen, flüssigkeitsdichten Einmalhandschuhen (z. B. nach DIN EN 455) empfohlen. Diese müssen in ausreichender Zahl vorhanden sein. Es sollten puderfreie, allergenarme Latexhandschuhe oder andere geeignete Handschuhe bevorzugt werden. Ist mit einer Durchnässung der Kleidung zu rechnen, sollten zudem flüssigkeitsdichte Schürzen getragen werden.

Besteht darüber hinaus bereits die Gefahr, dass potentiell infektiöses Material oder Flüssigkeiten verspritzt bzw. versprüht werdem, so sollte das Personal einen geeigneten Augen- oder Gesichtsschutz tragen. 
Als Ergänzung zur PSA ist zudem bei Verdacht auf eine durch Aerosol oder Tröpfchen übertragbare Erkrankung eine partikelfiltrierende Halbmaske $\mathrm{FFP}_{2}$ erforderlich.

Sollte eine hoch kontagiöse Infektionskrankheit bekannt sein, so muss die PSA ggf. um einen Ganzkörperschutz (z. B. Overall), Augenschutz und eine partikelfiltrierende Halbmaske $\mathrm{FFP}_{3}$ (umluftabhängig) oder sogar durch die Verwendung umluftunabhängiger Atemschutzgeräte ergänzt werden. Die folgende Übersicht stellt die in der GUV-R 2106 empfohlene Ausstattung von vorzuhaltenden »Infektionsschutz-Sets« dar.

\section{Empfohlener Inhalt eines »Infektions- schutz-Sets"}

Möglichst in Folienschutzbeuteln mit Snap-

Verschluss verpackt.

- 1 Schutzanzug

Overall mit Haube, möglichst mit integrierten Füßlingen. Dichtigkeit gegen Mikroorganismen nach DIN EN 14 126. Leistungsklasse entsprechend dem Schutzziel zu wählen.

- Ggf. Schutzkittel Knöchellang, Entfall bei Verwendung eines Overalls

- 1 Atemschutz Partikel filtrierende Halbmaske nach DIN EN 149, Schutzstufe $F F P_{2}$, gegebenenfalls $\mathrm{FFP}_{3}$, mit oder ohne Ausatemventil (keine OP-Masken)

- 2 Paar Schutzhandschuhe (Paar) Geprüft nach DIN EN 455, AQL 1, 5; mit extra langen Stulpen

- 1 Kopfhaube Entfällt bei Overallhaube

- 1 Schutzbrille z. B. nach DIN EN 166, mit Seitenschutz und indirekter Belüftung sowie Abdeckung im Augenbrauenbereich

- 2 Paar Überziehschuhe Bei Overall ohne Füßlinge oder Schutzkittel

- 2 Entsorgungsbeutel Kennzeichnung: Abfallgruppe $C$ Kunststoffsäcke (z. B. PE), mind. 0,08 mm Wandstärke

\subsection{Meldepflichtige Erkrankungen}

Gemäß $₫ 6$ des Infektionsschutzgesetzes (IfSG) sind namentlich folgende Erkrankungsfälle bei Krankheitsverdacht, Erkrankung oder Tod dem zuständigen Gesundheitsamt zu melden.

\section{Meldepflichtige Erkrankungsfälle}

- Botulismus

- Cholera

- Diphtherie

- Humaner spongiformer Enzephalopathie, außer familiär-hereditärer Formen

- Akuter Virushepatitis

- Enteropathisches hämolytisch-urämisches Syndrom (HUS)

- Virusbedingtes hämorrhagisches Fieber

- Masern

- Meningokokken-Meningitis oder-Sepsis

- Milzbrand

- Poliomyelitis: als Verdacht gilt jede akute schlaffe Lähmung, außer wenn traumatisch bedingt

- Pest

- Tollwut

- Typhus abdominalis/Paratyphus

- Erkrankung und Tod an einer behandlungsbedürftigen Tuberkulose, auch wenn ein bakteriologischer Nachweis nicht vorliegt

Weiterhin ist namentlich der Verdacht auf und die Erkrankung an einer mikrobiell bedingten Lebensmittelvergiftung oder an einer akuten infektiösen Gastroenteritis zu melden, wenn

- eine Person betroffen ist, die eine Tätigkeit im Sinne des $₫ 42$ Abs. 1 (Umgang mit Lebensmitteln) ausübt,

- zwei oder mehr gleichartige Erkrankungen auftreten, bei denen ein epidemischer Zusammenhang wahrscheinlich ist oder vermutet wird.

Zudem ist die Verletzung eines Menschen durch ein tollwutkrankes, - verdächtiges oder -ansteckungsverdächtiges Tier sowie die Berührung eines solchen Tieres oder Tierkörpers zu melden. 
Gemäß $₫ 8$ des IfSG (Auszug) sind folgende im Rettungsdienst relevante Personen zur Meldung verpflichtet:

1. Feststellende Arzt; in Krankenhäusern oder anderen Einrichtungen der stationären Pflege ist für die Einhaltung der Meldepflicht neben dem feststellenden Arzt auch der leitende Arzt, in Krankenhäusern mit mehreren selbständigen Abteilungen der leitende Abteilungsarzt, in Einrichtungen ohne leitenden Arzt der behandelnde Arzt verantwortlich

2. Leiter von Medizinaluntersuchungsämtern und sonstigen privaten oder öffentlichen Untersuchungsstellen einschließlich der Krankenhauslaboratorien

3. Tierarzt, bei Tieren, mit denen Menschen Kontakt gehabt hatten

4. Angehörige eines anderen Heil- oder Pflegeberufs, der für die Berufsausübung oder die Führung der Berufsbezeichnung eine staatlich geregelte Ausbildung oder Anerkennung erfordert

5. Verantwortliche Luftfahrzeugführer oder der Kapitän eines Seeschiffes

6. Leiter von Pflegeeinrichtungen, Justizvollzugsanstalten, Heimen, Lagern oder ähnlichen Einrichtungen

7. Heilpraktiker

Laut Absatz (2) des $\$ 8$ IfSG besteht die Meldepflicht nicht für Personen des Not- und Rettungsdienstes, wenn der Patient unverzüglich in eine ärztlich geleitete Einrichtung gebracht wurde. Die Meldepflicht besteht für die unter Nr. 4 bis 6 bezeichneten Personen nur, wenn ein Arzt nicht hinzugezogen wurde.

\subsection{Infektionstransport}

Die darüber hinaus notwendigen Hygiene- und Vorsichtsmaßnahmen richten sich nach der Einteilung der (Notfall-) Patienten in folgende Kategorien:

\section{Kategorie $\mathrm{A}$}

- Patienten ohne Anhalt für das Vorliegen einer Infektionserkrankung

\section{Kategorie B}

- Patienten mit bestehender Infektion, wobei diese jedoch nicht durch beim Transport übli- che Kontakte übertragbar ist, z. B. auch Virushepatitis, HIV-Infektion ohne klinische Zeichen des Vollbildes Aids, Tuberkulose

- Ausgenommen sind Patienten mit offener Lungentuberkulose, sowie Patienten mit offenen und blutenden Wunden (s. Kategorie C oder D)

\section{Kategorie C-I}

- Patienten mit gesicherter Diagnose oder begründetem Verdacht auf eine kontagiöse Infektionskrankheit wie z. B. offene Lungentuberkulose, Meningokokken-Meningitis, Diphtherie, Milzbrand, Windpocken, generalisiertem Zoster, Cholera, Typhus, Tollwut

- Patienten mit multiresistenten Keimen wie z. B. MRSA, VRE

- Patienten mit akutem Erbrechen und/oder Ausscheidung dünnflüssiger Stühle

\section{Kategorie C-II}

- Patienten mit bloßem Verdacht auf eine Infektionskrankheit mit besonders gefährlichen Erregern wie z. B. hämorrhagisches Fieber (Lassa, Ebola), Pocken, Pest, Lungenmilzbrand oder SARS

\section{Kategorie D}

- Patienten mit einer besonderen Infektionsgefährdung z. B. durch Polytrauma, ausgedehnte Unfallverletzungen/Verbrennungen, Frühgeburt oder insbesondere Immunsuppression (z. B. manifeste Aids-Erkrankung, Leukopenie (<500 Neutrophile), Agranulozytose, Organtransplantation)

\section{(D) Wichtig}

Bei Patienten der Kategorie A und B sind keine über das normale Maß hinausgehenden hygienischen Maßnahmen notwendig, weil nicht von einer erhöhten Gefährdung für Patient und Personal ausgegangen werden muss.

\section{Durchführung von Patiententransporten}

Bei der Durchführung von Transporten sind auch im Hinblick auf die Infektionsgefährdung primär Notfalltransporte von elektiven Krankentransporten $\mathrm{zu}$ unterscheiden. Generell sind bei Patienten der Kategorie A und B keine über die Standardhy- 
giene- und Vorsichtsmaßnahmen hinausgehenden Vorkehrungen erforderlich.

\section{Notfalltransporte}

- Eine Infektion oder Infektionsgefährdung des Patienten ist in der Regel nicht bekannt.

- Erforderliche Maßnahmen zur Desinfektion können also ggf. erst im Nachhinein durchgeführt werden.

- Standardhygiene und Vorsichtsmaßnahmen sollten bei jedem Transport beachtet werden.

\section{Krankentransporte}

\section{Kategorie A}

- Standardhygiene und Vorsichtsmaßnahmen

- Nach dem Transport laufende Desinfektion gemäß Hygieneplan

\section{Kategorie B}

- Standardhygiene und Vorsichtsmaßnahmen

- Nach dem Transport laufende Desinfektion gemäß Hygieneplan

- Bedenke Hygiene- und Vorsichtsmaßnahmen bezogen auf Herkunft des infektiösen Materials (• Tab. 2.6)

- Scheuer-Wisch-Desinfektion horizontaler Flächen und ggf. potenziell kontaminierter vertikaler Flächen

\section{Kategorie C-I und C-II}

- Im Vorfeld Abklärung erforderlicher besonderer Schutzmaßnahmen durch die Rettungsleitstelle und Dokumentation der wichtigsten Informationen (Erreger, Zustand des Patienten, Ansprechpartner).

- Tab. 2.6. Hygiene- und Vorsichtsmaßnahmen bezogen auf Herkunft des infektiösen Materials

\begin{tabular}{|c|c|c|c|}
\hline $\begin{array}{l}\text { Mögliche Erkrankung/ } \\
\text { Erreger }\end{array}$ & $\begin{array}{l}\text { Infektiösität } \\
\text { durch }\end{array}$ & Übertragungsweg & Schutzmaßnahmen \\
\hline $\begin{array}{l}\text { Hepatitis B und C, } \\
\text { HIV }\end{array}$ & $\begin{array}{l}\text { Blut bzw. } \\
\text { Körperflüs- } \\
\text { sigkeiten }\end{array}$ & $\begin{array}{l}\text { Parenteraler Kontakt } \\
\text { (Verletzung) }\end{array}$ & $\begin{array}{l}\text { - Standardhygiene- und Vorsichtsmaßnahmen } \\
\text { - Schutz vor parenteralem Kontakt mit in- } \\
\text { fektiösem Material (z. B. blutkontaminierte } \\
\text { Gegenstände) }\end{array}$ \\
\hline $\begin{array}{l}\text { Meningokokken- } \\
\text { Meningitis, Diphtherie, } \\
\text { Scharlach, Windpocken, } \\
\text { Masern, Röteln }\end{array}$ & $\begin{array}{l}\text { Respiratori- } \\
\text { sches Sekret }\end{array}$ & $\begin{array}{l}\text { Naher Kontakt not- } \\
\text { wendig (Tröpfchen } \\
\text { und Schleimhaut- } \\
\text { kontakt) }\end{array}$ & $\begin{array}{l}\text { - Standardhygiene- und Vorsichtsmaßnahmen } \\
\text { - Schutz vor direktem/indirektem Kontakt } \\
\text { mit infektiösem Material (z. B. Schleim und } \\
\text { Sekret der oberen Atemwege) } \\
\text { - Mund-Nasen-Schutz für Patienten und Per- } \\
\text { sonal }\end{array}$ \\
\hline $\begin{array}{l}\text { Offene Lungentuber- } \\
\text { kulose }\end{array}$ & Aerosole & $\begin{array}{l}\text { Abhängig vom Aus- } \\
\text { maß des Aerosolkon- } \\
\text { taktes, kein Risiko } \\
\text { bei geschlossener } \\
\text { Beatmung! }\end{array}$ & $\begin{array}{l}\text { - Standardhygiene- und Vorsichtsmaßnahmen } \\
\text { - Schutz vor Inhalation infektiöser Aerosole } \\
\text { - TB-Maske für Patienten ggf. für Personal }\end{array}$ \\
\hline $\begin{array}{l}\text { Gastro-/Enteritis durch } \\
\text { Salmonellen, Shigellen, } \\
\text { Hepatitis A und E, Nor- } \\
\text { walk-like-Virus }\end{array}$ & Stuhl & $\begin{array}{l}\text { Orale Aufnahme des } \\
\text { Erregers, kein Risiko } \\
\text { ohne Stuhlkontakt! }\end{array}$ & $\begin{array}{l}\text { - Standardhygiene- und Vorsichtsmaßnahmen } \\
\text { - Schutz vor direktem und indirektem Kon- } \\
\text { takt mit infektiösem Material wie Stuhl, ggf. } \\
\text { Erbrochenes }\end{array}$ \\
\hline $\begin{array}{l}\text { Methicilinresistente } \\
\text { S. aureus/MRSA, Vanco- } \\
\text { mycin resistente Entero- } \\
\text { kokken/VRE u. Ä. }\end{array}$ & $\begin{array}{l}\text { Kolonisation } \\
\text { mit multi- } \\
\text { resistenten } \\
\text { Keimen }\end{array}$ & $\begin{array}{l}\text { Kein Risiko für } \\
\text { gesundes Personal } \\
\text { Kein Risiko durch in- } \\
\text { fizierte Wunde ohne } \\
\text { Wundkontakt }\end{array}$ & $\begin{array}{l}\text { - Standardhygiene- und Vorsichtsmaßnahmen } \\
\text { - Bei nasaler Besiedelung Gesichtsmaske für } \\
\text { den Patienten! }\end{array}$ \\
\hline
\end{tabular}


- Sind Erreger oder Erkrankung sowie das notwendige Verhalten Leitstelle oder Fahrzeugbesatzung nicht geläufig, Rücksprache mit dem zuständigen Desinfektor oder der Hygienefachkraft.

- Wenn möglich sollte kein RTW, sondern im Idealfall ein eigens dafür vorgesehener KTW verwendet werden.

- Wenn möglich Überprüfung des Immunstatuts der Besatzung, ggf. Rücksprache mit Betriebsarzt.

- Weitergabe aller Informationen der Rettungsleitstelle an die Fahrzeugbesatzung.

- Ggf. Information des zuständigen Desinfektors/Hygienefachkraft über den Transport und Abstimmung weiterer Maßnahmen.

- Spezielle Vorkehrungen ( $\bullet$ Übersicht).

- Vorgehen gemäß Empfehlungen des behandelnden Arztes entsprechend dem Immunstatus des Patienten.

- Tragen erforderlicher Schutzausrüstung (z. B. Einmaloverall, Überschuhe, Schutzhaube, Mund-Nasen-Schutz, Einmalhandschuhe).

- Nach dem Transport Desinfektion des Rettungsmittels, ggf. in Rücksprache mit dem zuständigen Desinfektor.

\section{Spezielle Vorkehrungen bei elektiven} Infektionstransporten

- Detaillierte Information der Fahrzeugbesatzung sowie des Zielkrankenhauses

- Wenn möglich speziell vorgehaltenes Rettungsmittel verwenden, ggf. Ausräumung des Patientenraumes

- Fahrerabteil getrennt halten - Zwischenfenster schließen und Verständigung sicherstellen

- Lüftung im Fahrzeug auf keinen Fall auf Innenbelüftung umschalten

- Fahrer bleibt dem Patienten möglichst fern, öffnet und schließt Türen

- Ggf. Tragen von erforderlicher Schutzkleidung bzw. Vollschutz

- Mitnahme von Material für Desinfektionsmaßnahmen (geeignete Desinfektionsmittel, Plastiksäcke zur luftdichten Entsorgung gebrauchter Materialien sowie der Kleidung)

- Umgehende Desinfektion von Blut, Stuhl, Harn, Sputum oder anderen Sekreten

- Transportunterbrechung nur bei vitaler Bedrohung des Patienten

- Wechsel der Einsatzbekleidung und Duschen der Einsatzkräfte nach dem Einsatz

- Desinfektion des Rettungsmittels nach dem Einsatz, ggf. in Rücksprache mit dem zuständigen Desinfektor

\section{Kategorie D}

- Bei elektiven Transporten vorab Desinfektion des eingesetzten Rettungsmittels

- Vorgehen gemäß Empfehlungen des behandelnden Arztes entsprechend dem Immunstatus des Patienten

- Ggf. Bereitstellung erforderlicher Schutzausrüstung für den Patienten

- Nach dem Transport laufende Desinfektion gemäß Hygieneplan

\section{Vorgehen bei Patienten mit Methicillin- resistenten Staphylococcus aureus (MRSA)}

In Deutschland stieg in den vergangenen 10 Jahren die MRSA-Prävalenz von $2 \%$ auf ca. $23 \%$ aller S.-aureus-Isolate. Rettungsdienst und Krankentransport stellen die Schnittstelle zwischen klinischer Versorgung mit notwendigen strengen Hygienemaßnahmen zur Bekämpfung von MRSA, und dem ambulanten Bereich, wo die Anwendung der Standard-Hygienemaßnahmen ausreichend ist, dar.

\section{(? Wichtig}

Die Weiterverbreitung von MRSA wird hauptsächlich durch Missachtung von StandardHygienemaßnahmen, z. B. nicht ausreichende hygienische Händedesinfektion, verursacht!

Da es gerade an dieser Schnittstelle oft zu großen Irritationen über die beim Transport von MRSAPatienten erforderlichen Maßnahmen kommt, hier einige Anmerkungen:

- Rettungsdienstpersonal ist beim Transport von MRSA-besiedelten Patienten nicht stärker in- 
fektionsgefährdet als beim Transport anderer Patienten, solange die Standard-Hygiene- und Vorsichtsmaßnahmen eingehalten werden.

- Ein spezielles Einsatzfahrzeug für Infektionstransporte sowie die Verwendung eines sog. Vollschutzanzuges ist nicht erforderlich.

- Rettungsdienstpersonal und Zieleinrichtung sind über die MRSA-Besiedelung $\mathrm{zu}$ informieren.

- Übertragung ist über Hautkontakt und aerogen möglich.

- Etwaige Wunden sollten vor dem Transport frisch verbunden und abgedeckt werden.

- Bei Besiedlung der Atemwege trägt der Patient einen Mund-Nasen-Schutz.

- Wenn möglich sollte der Patient vor Transportbeginn eine hygienische Händedesinfektion durchführen.

- Das Tragen von Einmalhandschuhen und eines zusätzlichen Schutzkittels zur Verhinderung einer Kontamination der Arbeitskleidung bei allen Versorgungsmaßnahmen ist ausreichend.

- Das Tragen eines Mund-Nasen-Schutzes ist im Krankentransport und Rettungsdienst sinnvoll, da hier auf engstem Raum gearbeitet wird: In jedem Fall ist dieser bei endotrachealem Absaugen oder notwendigem Verbandswechsel zu tragen, um eine aeroge Übertragung zu vermeiden.

- Nach Transportbeendigung ist eine hygienische Händedesinfektion durchzuführen.

- Desinfektionsmaßnahmen nach dem Transport können grundsätzlich mit einem Desinfektionsmittel zur »laufenden Desinfektion« laut Hygieneplan in Form einer Scheuer-WischDesinfektion durchgeführt werden.

\section{Literatur}

Arbeitskreis »Krankenhaus- \& Praxishygiene« der AWMF: Hygiene in Klinik und Praxis, Hygienemaßnahmen beim Patiententransport; 3. Auflage, mhp, Wiesbaden 2004, S. $214 \mathrm{ff}$

Bundesärztekammer: Zur Frage der Verbindlichkeit von Richtlinien, Leitlinien, Empfehlungen und Stellungnahmen, 01.06.1998, http://www.bundesaerztekammer. de/30/Richtlinien/90Verbindlich.html

Bundesverband der Unfallkassen, GUV-R 2106 (bisher GUV 27.10), Regeln für Sicherheit und Gesundheitsschutz:
GUV-Regel Benutzung von persönlichen Schutzausrüstungen im Rettungsdienst; Ausgabe Oktober 2005

Buschhausen-Denker G, Möller PM, Technische Regeln für Biologische Arbeitsstoffe-TRBA 250, Neue Anforderungen an den Arbeitsschutz im Rettungsdienst? Notfall \& Rettungsmedizin, (7) 5, August 2004, 337-342

Garner JS (1996) CDC Guideline for Isolation precautions in Hospital. Infect Control HospEpidemiol. 16: 53-80

Gesetz zur Verhütung und Bekämpfung von Infektionskrankheiten beim Menschen (Infektionsschutzgesetz - IfSG), Artikel 1 des Gesetzes zur Neuordnung seuchenrechtlicher Vorschriften - (Seuchenrechtsneuordnungsgesetz - SeuchRNeuG vom 20. Juli 2000)

Hauptverband der gewerblichen Berufsgenossenschaften (HVBG), Technische Regeln für Biologische Arbeitsstoffe im Gesundheitswesen und in der Wohlfahrtspflege, TRBA 250, Oktober 2003

Kommission für Krankenhaushygiene und Infektionsprävention beim Robert-Koch-Institut (RKI), Anforderungen an die Hygiene bei der Reinigung und Desinfektion von Flächen. Bundesgesundheitsbl - Gesundheitsforsch - Gesundheitsschutz 2004, 47:51-61

Robert-Koch-Institut, Deutsch-Österreichische Empfehlungen zur HIV-Postexpositionsprophylaxe (HIV-PEP), Stand November 2004

Robert-Koch-Institut, Epidemiologischen Bulletin des RKI, $1 / 2000$, S. $1-2$

Robert-Koch-Institut, Empfehlungen der Ständigen Impfkommission (STIKO); Stand: Juli 2006; Epidemiologisches Bulletin 30/2006, Erscheinungsdatum: 31.07.2006

Schnelle R, Nadelstich-Sofortmaßnahmen-Set: Eine Ergänzung der RTW-Ausstattung. Rettungsdienst 29 (11); November 2006, 113

\section{Weiterführende Internetadressen}

Arbeitskreis »Krankenhaus- \& Praxishygiene» der AWMF: http://www.AWMF-online.de

Berufsgenossenschaft für Gesundheitsdienst und Wohlfahrtspflege, gesetzliche Unfallversicherung für nichtstaatliche Einrichtungen im Gesundheitsdienst und in der Wohlfahrtspflege

Bundesanstalt für Arbeitsschutz und Arbeitsmedizin (BAuA) http://www.baua.de

Informationen über MRSA: http://www.mrsa-net.org

Robert-Koch-Institut: http://www.rki.de

http://www.bgw-online.de

http://www.desinfektor.net

http://www.unfallkassen.de

http://www.nadelstichverletzung.de 